\title{
Two-beam photoacoustic phase measurement of the thermal diffusivity of a Gd-doped bulk YBCO superconductor
}

\author{
M. Aravind, P. C. W. Fung, S. Y. Tang, and H. L. Tam \\ Department of Physics, The University of Hong Kong, Hong Kong
}

(Received 8 May 1995; accepted for publication 4 January 1996)

\begin{abstract}
The two-beam photoacoustic phase measurement was applied to measure quantitatively the thermal diffusivity $\left(\alpha_{s}\right)$ of a ceramic bulk high- $T_{c}$ superconductor. Neglecting the effects of thermal dilation, and thermoelastic bending was proved valid in accordance with our composite piston model for the chosen experimental conditions. It was found that $\alpha_{s}$ shows different features at the onset and offset temperatures corresponding to the normal-superconducting (NS) transition. A dip was seen at the resistivity transition onset temperature and a cusp at the offset temperature where the electrical resistance disappears. The presence of the cusp at the offset temperature is proposed to be related to weak coupling between superconducting grains. Our studies indicate that the two-beam phase measurement is a very sensitive method for superconductor characterization and NS transition detection. The experimental results also confirm the presence of a large energy gap and strong electron-phonon coupling mechanism in the YBCO superconductor. (C) 1996 American Institute of Physics. [S0034-6748(96)00504-0]
\end{abstract}

\section{INTRODUCTION}

Resistivity-temperature $(R-T)$ and susceptibilitytemperature $(\chi-T)$ measurements, which probe the two basic aspects of superconductors-zero resistivity and perfect diamagnetism, respectively - are most common in superconductor characterization. However, in $R-T$ measurements if minority phases that are superconducting form paths in the sample, then an indication of zero resistance of the sample would lead us to the erroneous conclusion that the bulk is superconducting. Also, in $\chi-T$ measurements if a particle of the majority phase is shielded completely by a layer of superconducting minority phase on its surface then the transition of the latter might be mistakenly identified as being that of the whole particle volume. ${ }^{1}$

In view of the drawbacks in $R-T$ and $\chi-T$ measurements, various other methods have been adapted for superconductor characterization. Application of contactless methods, such as photoacoustic (PA) $)^{2,3}$ and photothermal deflection (PTD) techniques, ${ }^{4}$ has added a new dimension to normal-superconducting (NS) phase transition detection. They provide more direct, sensitive, economical, and convenient studies of the bulk features of any form of sample without special treatment. In this paper, we present our results on the thermal diffusivity measurement of a high- $T_{c}$ superconductor ceramic sample using a two-beam photoacoustic phase measurement. ${ }^{5}$ Advantages of our method over that of Song et al., ${ }^{2}$ who used a conventional single beam photoacoustic method, are elucidated in Sec. II. We have recorded the temperature dependence of thermal diffusivity of the sample and found different features corresponding to the NS transition onset and offset. These findings bear out the success of the PA method in superconductor characterization and NS transition detection.

\section{TWO-BEAM PHOTOACOUSTIC PHASE MEASUREMENT}

In the photoacoustic measurement of the diffusivity of solids, ${ }^{6}$ the sample, with a backing material at its rear, is encased in a closed cell containing a gas and a sensitive microphone. The front surface of the sample is illuminated with a chopped monochromatic light beam. Light is absorbed by the sample periodically and is converted into thermal energy by a random deexcitation process. Thermal diffusion from the sample causes a periodic temperature variation in a thin layer of gas at the sample-gas boundary, which results in a pressure oscillation in the cell. This pressure oscillation, with the same period but not necessarily the same phase as the chopped light beam, induces an analog signal after it is picked up by the microphone. The phase difference is a consequence of the finite relaxation time in deexcitation. The expression for the pressure variation for an optically thick sample is well established: ${ }^{7}$

$$
p_{F}=\frac{\gamma P_{0} I_{F}}{2 T_{0} I_{g} K_{s}} \sqrt{\frac{\alpha_{g}}{\alpha_{s}}} \frac{g+\operatorname{coth}\left(l_{s} \sigma_{s}\right)}{I+g \operatorname{coth}\left(l_{s} \sigma_{s}\right)} \frac{1}{\sigma_{s}^{2}},
$$

where $l$ is the thickness, $\alpha$ the thermal diffusivity, and $K$ the thermal conductivity, with the subscripts $g$ and $s$ symbolizing that of the gas and the solid sample, respectively. $T_{0}$ and $P_{0}$ are the static temperature and pressure of the gas, respectively, and $\gamma$ is the ratio of the heat capacities $C_{p} / C_{v}$ of the gas. $I_{F}$ stands for the power of light absorbed at the front surface. Similar effect could be detected in the case of rearsurface illumination and the pressure variation is expressible in the form ${ }^{7}$

$$
p_{R}=\frac{\gamma P_{0} I_{R}}{2 T_{0} l_{g} K_{s}} \sqrt{\frac{\alpha_{g}}{\alpha_{s}}} \frac{1}{\sinh \left(l_{s} \sigma_{s}\right)\left(1+\frac{g}{\tanh \left(l_{s} \sigma_{s}\right)}\right)} \frac{1}{\sigma_{s}^{2}},
$$

where the complex quantity is

$$
\sigma_{s}=(1+i) \sqrt{\frac{\pi f}{\alpha_{s}}},
$$

and $g$ is the ratio of thermal effusivities $\left(e=K / \alpha^{1 / 2}\right)$ between the backing $\left(e_{b}\right)$ and the sample $\left(e_{s}\right)$, i.e., 


$$
g=\frac{e_{b}}{e_{s}}=\frac{K_{b}}{K_{s}} \sqrt{\frac{\alpha_{s}}{\alpha_{b}}}
$$

while $f$ is the chopping frequency.

By visualizing Eqs. (1)-(4), we notice that if only one beam (either the front or the rear beam) is used, the signal depends on many parameters among which the power $I_{F}$ (or $I_{R}$ ) of the absorbed light and the static pressure $P_{0}$ are difficult to measure. Although lots of parameters are eliminated by using signal magnitudes in both the front and the rear cases, namely,

$$
\frac{\left|\delta p_{F}\right|}{\left|\delta p_{R}\right|}=\frac{I_{F}}{I_{R}} \sqrt{g^{2}\left(\cosh ^{2} x-\cos ^{2} x\right)+\left(\cosh ^{2} x-\sin ^{2} x\right)+g \sinh 2 x}
$$

where

$$
x=l_{s} \sqrt{\frac{\pi f}{\alpha_{s}}}
$$

the powers of the absorbed light in the front and the rear surface are in general different. (This is due to the different surface conditions and the instability of the light source). However, $I_{F} / I_{R}$ is still involved.

However, if the relative phases $\Delta \Psi=\Psi_{F}-\Psi_{R}$ of the signals from the front and the rear surfaces are measured, we can deduce $x$, hence $\alpha_{s}$, regardless of the absorbed powers. Mathematical verification of this idea is presented below.

Phase angles of the photoacoustic signal relative to the chopped incident' light for the cases of front-surface illumination $\left(\Psi_{F}^{\prime}\right)$ and rear-surface illumination $\left(\Psi_{R}^{\prime}\right)$ can be deduced from Eqs. (1)-(4) as follows:

$$
\begin{aligned}
& \tan \psi_{F}^{\prime}=\frac{\sin ^{2} x(1+g \tanh x)(g+\tanh x)}{\tan x\left(1-g^{2}\right) \sin ^{2} x}, \\
& \tan \psi_{R}^{\prime}=\frac{(g+\tanh x)}{\tan x(1+g \tanh x)} .
\end{aligned}
$$

In practice, an additional phase $\Psi_{0}$, which depends on the cell design and other experimental components being the same for the front and rear cases, is superimposed on both $\Psi_{F}^{\prime}$ and $\Psi_{R}^{\prime}$,

$$
\begin{aligned}
& \psi_{F}=\psi_{F}^{\prime}+\psi_{0}, \\
& \psi_{R}=\psi_{R}^{\prime}+\psi_{0}
\end{aligned}
$$

but the relative phase is independent of $\Psi_{0}$ :

$$
\Delta \psi=\psi_{F}-\psi_{R}=\left(\psi_{F}^{\prime}+\psi_{0}\right)-\left(\psi_{R}^{\prime}+\psi_{0}\right)=\psi_{F}^{\prime}-\psi_{R}^{\prime} .
$$

After simple algebrical steps we obtain

$$
\tan (\Delta \psi)=\tan x \frac{g+\tanh x}{1+g \tanh x} .
$$

It is clear that if $\Delta \Psi$ is measured and the value of $g$ is known, $x$ could be solved either graphically or numerically. The thermal diffusivity of the solid sample $\alpha_{s}$ can then be deduced from Eq. (6) from which the values of $f$ and $l_{s}$ could be easily measured. The merits of two-beam phase measurement over single-beam measurement are obvious: Owing to the independence of $\Delta \Psi$ on the power absorption of the sample surface, the two-beam phase measurement al- lows $\alpha_{s}$ of the sample to be measured directly. A further simplification of Eq. (10) can be done by using air backing. Since the effusivity of air is negligible compared to that of solid samples, we could set $g=0$ in Eq. (10) if the backing material used is air, and Eq. (10) simplifies to Pessoa's result: ${ }^{5}$

$$
\tan (\Delta \psi)=\tan x \tanh x
$$

\section{EFFECTS OF THERMAL DILATION AND THERMOELASTIC BENDING IN TWO-BEAM PHASE MEASUREMENT}

Until now, we have been considering the contribution of the acoustic piston ${ }^{6}$ (i.e., Rosencwaig and Gersho's consideration) to $\Delta \Psi$ only. Basically, two more mechanisms are involved. One of them is the thermal dilation effect, ${ }^{7,8}$ which originates from the periodic expansion of the sample along its thickness after the sample was heated up by the chopped light beam. The significance of this effect depends on the mean sample temperature. Another effect one has to take into account is thermoelastic bending, ${ }^{7,9}$ which is attributed to transverse thermal expansion and the existence of a nonzero temperature gradient throughout the thickness of the sample.

Rousset et al. ${ }^{9}$ have derived the expression for the displacement of a point $(r, z)$ in cylindrical coordinates in the sample from its equilibrium position due to heating of the sample by the light source in the case of rear illumination. Particularly in the $z$ direction, the expression for the stated displacement is as follows:

$$
\begin{aligned}
u_{z}^{R}(r, z)= & a_{T}\left\{\frac{6\left(R^{2}-r^{2}\right)}{l_{s}^{3}} M_{T}+\frac{1+\nu}{1-\nu} \int_{l_{s / 2}}^{z} T_{s}^{R} d z\right. \\
& \left.-\frac{\nu}{1-\nu}\left[\frac{12 M_{T}}{l_{s}^{3}}\left(z^{2}-\frac{l_{s}^{2}}{4}\right)+\frac{2 N_{T}}{l_{s}}\left(z-\frac{l_{s}}{2}\right)\right]\right\}
\end{aligned}
$$

with

$$
M_{T}=\int_{-l_{s / 2}}^{l_{s / 2}} z T_{s}^{R} d z, \quad N_{T}=\int_{-l_{s / 2}}^{l_{s / 2}} T_{s}^{R} d z,
$$

where $a_{T}$ is the linear thermal expansion coefficient and $\nu$ is its Poisson ratio. The temperature distribution $T_{s}^{R}$ inside a thermally thick sample is given by 


$$
T_{s}^{R}=\frac{I_{0}^{R} \cosh \left[\sigma_{s}\left(z-\frac{l_{s}}{2}\right)\right]}{2 K_{s} \sigma_{s} \sinh \left(\sigma_{s} l_{s}\right)} .
$$

By modifying Rousset's expression above to the case of front-surface illumination, we get

$$
\begin{aligned}
u_{z}^{F}(r, z)= & a_{T}\left\{\frac{6\left(R^{2}-r^{2}\right)}{l_{s}^{3}} M_{T}+\frac{1+\nu}{1-\nu} \int_{l_{s / 2}}^{z} T_{s}^{F} d z\right. \\
& \left.-\frac{\nu}{1-\nu}\left[\frac{12 M_{T}}{l_{s}^{3}}\left(z^{2}-\frac{l_{s}^{2}}{4}\right)+\frac{2 N_{T}}{l_{s}}\left(z+\frac{l_{s}}{2}\right)\right]\right\},
\end{aligned}
$$

where

$$
T_{s}^{F}=\frac{I_{0}^{F} \cosh \left[\sigma_{s}\left(z+\frac{l_{s}}{2}\right)\right]}{2 K_{s} \sigma_{s} \sinh \left(\sigma_{s} l_{s}\right)} .
$$

In Eqs. (12) and (14) we noticed that only the first term is $r$ dependent and represents the thermoelastic bending effect. The other terms are $r$ independent and represent the effect of thermal dilation. So the thermoelastic bending effect is de- scribed by a quantity of the order of $R^{2} / l_{s}^{2}$ times larger than the thermal dilation effect, provided that the sample size satisfies $R>l_{s}$. Hence the second and latter terms in Eq. (14) can be neglected if $\left(R \gg l_{s}\right)$.

We can construct a composite piston as a sum of the acoustic piston (by RG) and thermoelastic bending

$$
p_{F, R}=\delta p_{F, R}^{R G}+\delta p_{F, R}^{T B},
$$

where $\delta p_{F, R}^{R G}$ are given by Eqs. (1) and (2), respectively, and

$$
\begin{aligned}
\delta p_{F, R}^{T B}= & \frac{\gamma P_{0}}{V_{0}} \int_{0}^{R} 2 \pi r u_{z}^{F, R}\left(r, \frac{l_{s}}{2}\right) d r \\
= & (+,-) \frac{3}{2} \frac{\gamma P_{0}}{l_{g}} \frac{a_{T} I_{0}^{F, R} R^{2}}{K_{s} \sigma_{s}^{3} l_{s}^{3}} \\
& \times \frac{\left(\frac{\sigma_{s} l_{s}}{2} \sinh \left(\sigma_{s} l_{s}\right)-\cosh \left(\sigma_{s} l_{s}\right)+1\right)}{\sinh \left(\sigma_{s} l_{s}\right)} .
\end{aligned}
$$

By separating the real and imaginary parts in Eq. (17) and extracting the phase angles for both cases of front- and rearsurface illumination, we get

$\tan \psi_{F}^{\prime}=\frac{b(\cosh x-\cos x)[(\sinh x+\sin x)-x(\cosh x+\cos x)]-a \sinh x \cosh x}{b(\cosh x-\cos x)(\sinh x-\sin x)-a \sin x \cos x}$,

$\tan \psi_{R}^{\prime}=\frac{b(\cosh x-\cos x)[(\sinh x+\sin x)-x(\cosh x+\cos x)]+a \sinh x \cos x}{b(\cosh x-\cos x)(\sin x-\sinh x)+a \sin x \cosh x}$,

where

$$
a=\frac{1}{T_{0}} \sqrt{\frac{\alpha_{g}}{\alpha_{s}}}, \quad b=\frac{3 a_{T}}{2 x}\left(\frac{R}{l_{s}}\right)^{2}
$$

The relative phase has been found to be expressed by

$$
\tan \Delta \psi=\frac{a \sin x \sinh x+b\left[x \sin x(\cos x+\cosh x)+\sinh ^{2} x-2 \sin x \sinh x-\sin ^{2} x\right]}{a \cos x \cosh x+b\left[x \sinh x(\cos x-\cosh x)+\cosh ^{2} x-2 \cos x \cosh x+\cos ^{2} x\right]}
$$

in which terms containing $b^{2}$ in the denominator have been neglected. This assumption is valid for solids due to the small values of $a_{T}$ ( $a_{T}$ is of the order of $10^{-5} \mathrm{~K}^{-1}$ in solids). We observed from Eq. (19) that terms (in the denominator or associated numerator) with the coefficient " $a$ " pertain to the acoustic piston effect while terms associated with the coefficient " $b$ " arise from the effect of thermoelastic bending.

It is noteworthy that in Eq. (19), $\Delta \Psi$ is independent of the power absorbed by the sample. This property allows us to use a rather large power light source to increase the signalto-noise ratio, without increasing the relative contribution of the thermoelastic bending effect to our measurement. This is the principal reason that prompted us to use two-beam PA phase measurement.

\section{VALIDITY OF ASSUMPTIONS}

Before we proceeded to our measurements, we had to choose our chopping frequency $f$ properly so that the assumptions for the equations in Sec. II would be valid.

First, justification of the assumption that the sample is optically thick is trivial, as it is optically opaque. Second, if 
TABLE I. Effects of thermoelastic bending relative to the acoustic piston in two-beam photoacoustic phase measurement, $f=35 \mathrm{~Hz}$.

\begin{tabular}{ccccc}
\hline \hline Temp (K) & $\alpha_{s}\left(\mathrm{~cm}^{2} / \mathrm{s}\right)$ & $a\left(\mathrm{~K}^{-1}\right)$ & $b\left(10^{-5} \mathrm{~K}^{-1}\right)$ & $b / a$ \\
\hline \multirow{2}{*}{293} & 0.05 & 0.0190 & 20.47 & 0.011 \\
& 0.10 & 0.0130 & 28.95 & 0.022 \\
& 0.50 & 0.0061 & 64.75 & 0.106 \\
& 1.00 & 0.0043 & 91.57 & 0.213 \\
& 0.05 & 0.0066 & 20.47 & 0.031 \\
100 & 0.10 & 0.0047 & 28.95 & 0.062 \\
& 0.50 & 0.0021 & 64.75 & 0.309 \\
& 1.00 & 0.0015 & 91.57 & 0.610 \\
\hline \hline
\end{tabular}

the PA signal varies as $f^{q}$ (where $q$ is a constant) for $-1.5 \leqslant q$ $<-1$ then the sample could be assumed to be thermally thick. ${ }^{6}$ The stated condition is found to be so if the chopping frequency ranges from 5 to $50 \mathrm{~Hz}$ under our experimental conditions. Hence we chose $35 \mathrm{~Hz}$ as our chopping frequency. Finally, as the sample radius $R$ is 8 times its physical thickness $l_{s}$, from Eqs. (12) and (14), the effect of thermoelastic bending is of the order of 64 times stronger than that of thermal dilation.

In view of Eqs. (19) and (6), as the parameter $b$ depends on the choice of $f$, we have compared the relative contribution from the acoustic piston and thermoelastic bending to $\Delta \Psi$ by calculating the ratio $b / a$ at $f=35 \mathrm{~Hz}$ at $293 \mathrm{~K}$ (room temperature) and $100 \mathrm{~K}$, respectively, for several values of $\alpha_{s}$ ranging from 0.05 to $1 \mathrm{~cm}^{2} \mathrm{~s}^{-1}$. The results are tabulated in Table I. The effect of the acoustic piston is shown to always be dominating especially when $\alpha_{s}$ is small. For $\alpha_{s}<0.5$, we can neglect the effect of thermoelastic bending on $\Delta \Psi$, as the acoustic piston effect is two orders of magnitude larger than the thermoelastic bending effect. Then we can simply apply Eq. (11) instead of Eq. (19) for calculating $\alpha_{s}$.

To check the validity of our theoretical model we have used the same method to determine the thermal diffusivity of various metals-whole thermal diffusivity values are known. The result of this analysis is shown below in Table II. ${ }^{21}$

From Table II it can be seen that the experimental values agree quite well with the literature values and hence confirm the validity of the theoretical model we have used. In calculating the value of $\alpha$ for the metals, the thermoelastic effect was also taken into account in addition to the thermal diffusion effect.

Though the thermal expansion coefficient of metals and the superconductors are roughly the same, the effect of ther-

TABLE II. The thermal diffusivity values obtained for different metals using our theoretical model.

\begin{tabular}{lccc}
\hline \hline \multicolumn{1}{c}{ Sample } & $\begin{array}{c}\text { Thickness } \\
l(\mathrm{~mm})\end{array}$ & $\begin{array}{c}\text { Measured } \\
\alpha\left(\mathrm{cm}^{2} / \mathrm{s}\right)\end{array}$ & $\begin{array}{c}\text { Literature value } \\
\alpha\left(\mathrm{cm}^{2} / \mathrm{s}\right)\end{array}$ \\
\hline Aluminum & 0.55 & 0.82 & $0.84^{\mathrm{a}}$ \\
Copper & 1.00 & 1.13 & $1.12^{\mathrm{a}}$ \\
Iron & 0.50 & 0.22 & $0.20^{\mathrm{a}}$ \\
Lead & 0.50 & 0.21 & $0.23^{\mathrm{a}}$ \\
Silicon & 0.52 & 0.72 & $0.76^{\mathrm{b}}$ \\
\hline \hline
\end{tabular}

${ }^{\mathrm{a}}$ Reference 21.

${ }^{\mathrm{b}}$ Reference 22 .

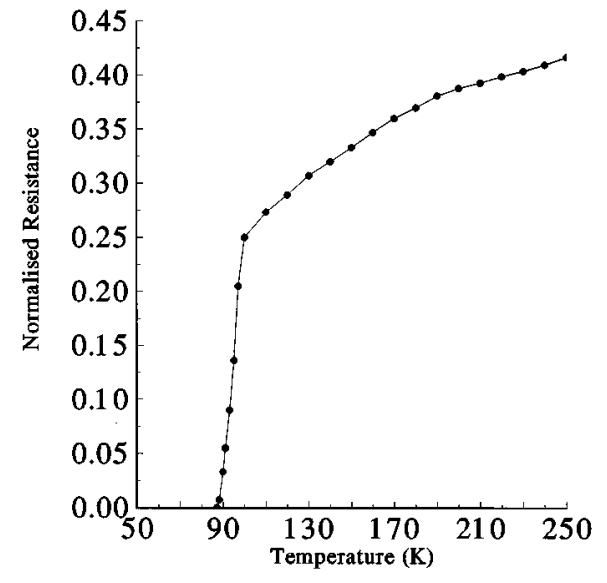

FIG. 1. Normalized $R-T$ curve of our $\mathrm{Y}_{0.6} \mathrm{Gd}_{0.4} \mathrm{Ba}_{2} \mathrm{Cu}_{3} \mathrm{O}_{7}$ sample. The resistance has been normalized to the value at room temperature.

moelastic bending is neglected in the case of the YBCO sample under our experimental conditions. This is justified as can be seen from the large value of $a$ in Table I as compared to the value of $b$.

\section{EXPERIMENTATION}

The bulk sample used was polycrystalline Gd-doped $\mathrm{YBCO}^{11,12}$ with a nominal composition of $\mathrm{Y}_{0.6} \mathrm{Gd}_{0.4} \mathrm{Ba}_{2} \mathrm{Cu}_{3} \mathrm{O}_{7}$, which was fabricated by simple solid state reaction. AR grade $\mathrm{Y}_{2} \mathrm{O}_{3}, \mathrm{Gd}_{2} \mathrm{O}_{3}, \mathrm{BaO}$, and $\mathrm{CuO}$ powders were mixed in the required ratio and ground thoroughly to a homogeneous mixture, which was preheated to $954{ }^{\circ} \mathrm{C}$ for $24 \mathrm{~h}$. Grinding and preheating were repeated again to promote homogeneity before the powder was pelletized and sintered at $964{ }^{\circ} \mathrm{C}$ for $24 \mathrm{~h}$ in flowing oxygen. Annealing was then performed at $300{ }^{\circ} \mathrm{C}$ for another $20 \mathrm{~h}$ in an oxygen-rich atmosphere.

The normalized resistance-temperature graph of the sample is shown in Fig. 1. The resistance values are normalized to the value of the resistance at room temperature, which is nearly $10^{-1} \Omega$. The measurement was taken in decreasing temperature. The transition onset was found to occur at $100 \mathrm{~K}$ while the offset at $88 \mathrm{~K}$.

The two-beam PA phase detection was performed in the following manner. The experimental setup is schematically depicted in Fig. 2. A Coherent-Innova 70, argon-ion laser (wavelength of $5145 \AA$, output power of $6 \mathrm{~W}$ ) was used as the light source. Periodic heating of the sample was achieved by chopping the laser beam, using a Stanford SR 540 mechanical chopper. The chopping frequency was chosen to be $35 \mathrm{~Hz}$, so that the assumptions for the equations in Sec. II are valid. The sample, in the form of a circular disc of radius $(R)$ $4 \mathrm{~mm}$ and thickness $\left(l_{s}\right) 0.5 \mathrm{~mm}$ was rigidly clamped on the sample chamber of a homemade PA cell such that the front surface of the sample is inside the chamber while the rear surface is opened to the bottom of the cryostat. The PA cell is of the Helmholtz resonator type ${ }^{13,14}$ with a resonant frequency of $484 \mathrm{~Hz}$ at room conditions. So, measurements were performed in nonresonant mode (as $f=35 \mathrm{~Hz}$ ). The PA cell was filled with dry nitrogen gas at a pressure of 0.58 bar. No special backing material was used. Cooling was achieved 


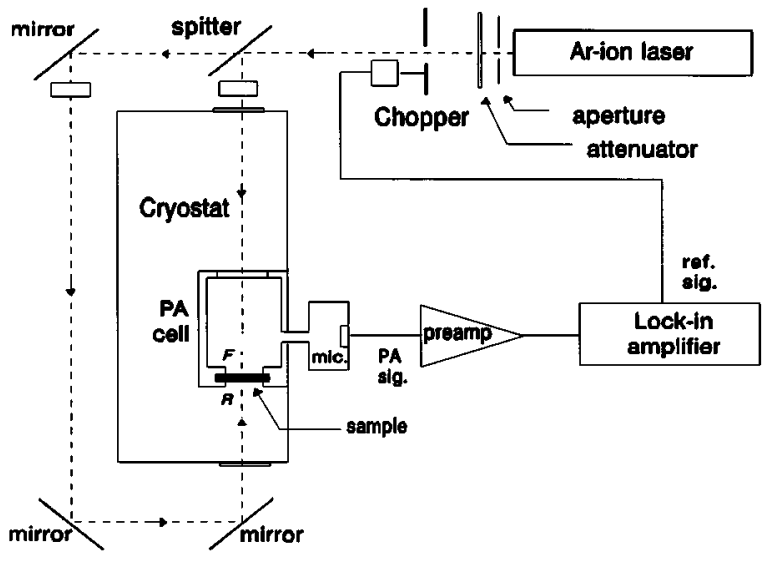

FIG. 2. Schematic diagram showing the experimental setup for two-beam photoacoustic phase measurement of thermal diffusivity.

by placing the PA cell inside a liquid nitrogen cryostat, which was equipped with a heater for temperature variation. The temperature was varied from room temperature down to 77 $\mathrm{K}$ and the value was measured by a calibrated platinum resistance thermometer with a resolution of $0.1 \mathrm{~K}$. The PA signal in the form of pressure variation was detected by an Electret microphone capsule KE 4-211-1 inside the microphone chamber, which was connected to the sample chamber by a capillary. The acoustic signal from the microphone was preamplified. The phase of this signal was compared with that of the reference signal from the chopper by a Stanford SR 530 phase lock-in amplifier. Due to apparatus limitation, front and rear illuminations were performed separately. Rearsurface measurement was performed using the mirror under the PA cell as shown in Fig. 2. All measurements were performed on a Newport RS4000 optical table to minimize spurious signals due to unwanted vibration.

\section{RESULTS AND ANALYSIS}

The sample was cooled in zero-magnetic field from 300 down to $87 \mathrm{~K}$. Measurement was performed for each degree decrease of temperature near the expected transition range. $\Psi_{F}$ and $\Psi_{R}$ were measured directly by the lock-in amplifier. $\Delta \Psi$ at various temperatures were obtained simply by subtracting $\Psi_{R}$ from $\Psi_{F}$. The values of $\Delta \Psi$ were fed into Eq. (11) and $x$ was solved. Thermal diffusivity of the sample $\alpha_{s}$ can be deduced easily from Eq. (6). The $\alpha_{s}-T$ plot is shown in Fig. 3.

We first noticed that $\alpha_{s}$ is of the order of magnitude 0.01 $\mathrm{cm}^{2} \mathrm{~s}^{-1}$. From Table I the ratio $b / a$ is smaller than 0.01 , which confirms the validity of applying Eq. (11) instead of Eq. (19), i.e., neglecting the effect of thermoelastic bending. Second, $\alpha_{s}$ showed a sharp cusp in the vicinity of $90 \mathrm{~K}$.

By comparing the $\alpha_{s}-T$ curve to the $R-T$ curve, we find that the cusp with a falling edge at $90 \mathrm{~K}$ in the $\alpha_{s}-T$ curve corresponds exactly to the resistive transition offset and the minor dip found at $100 \mathrm{~K}$ in the $\alpha_{s}-T$ curve correlates very well with the resistive transition onset. The falling edge in the cusp was predicted but not observed by Issac et al., ${ }^{15}$ who adopted single-beam PA measurement on $\mathrm{YBa}_{2} \mathrm{Cu}_{3} \mathrm{O}_{7}$.

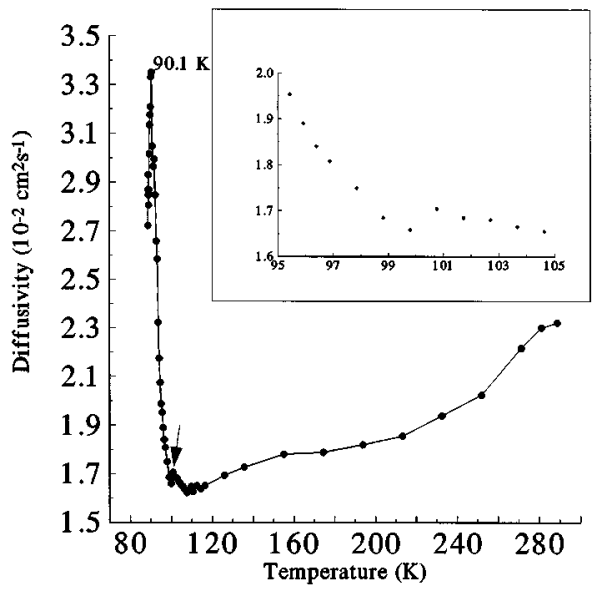

FIG. 3. $\alpha_{s}-T$ curve of our $\mathrm{Y}_{0.6} \mathrm{Gd}_{0.4} \mathrm{Ba}_{2} \mathrm{Cu}_{3} \mathrm{O}_{7}$ sample. The insert shows the curve in expanded scale in temperature near transition onset.

The result was seen by Peralta et al. ${ }^{23}$ who used photopyroelectric impulse response methods on similar samples.

We believe that the minor dip in $\alpha_{s}$ at the resistivity transition onset temperature is a result of the abrupt increase in the electron specific heat ${ }^{16,17}$ (due to the onset of superconductivity in individual grains). This can be visualized from the definition of thermal diffusivity viz. $\alpha_{s}=K_{s} / \rho_{s} C_{p}$ in which $C_{p}$ is the total (electrons and lattice) isobaric heat capacity.

Besides we find that the increase in $\alpha$ below $T_{c}$ must be due to the increase in the mean-free path of phonons. ${ }^{18} \mathrm{Be}-$ low $T_{c}$ the charge carriers condense to form Cooper pairs which do not scatter phonons, and as a result the mean-free path of phonons will increase. It is seen that below $T_{c}$, for high temperature YBCO samples, the thermal conductivity increases gradually ${ }^{18}$ though the specific heat decreases rapidly ${ }^{19}$ with decreasing temperature. These two effects cause the increase in the thermal diffusivity below $T_{c}$ to be very sharp. The sharp increase in the thermal diffusivity marks the onset of superconductivity. There is a competition between the rapidly diminishing electronic component of heat transport and the increasing phononic component. The cusp in the $\alpha_{s}-T$ curve is a result of this competition and coincides very well with the resistive transition offset temperature.

The increase in the thermal diffusivity is seen to be very steep and this may be due to the presence of a large energy gap in the superconductor. When the gap is larger the electrons condense more rapidly with decreasing temperature and the scattering of phonons by electrons will decrease. The cusp is seen at not too low a temperature as compared to the transition onset temperature, this predicts a strong electronphonon coupling. The cusp shifts to a temperature nearer the transition temperature for a coupling strength greater than the BCS one. ${ }^{20}$ The falling edge of the cusp is due to the decrease of the phonon population at very low temperatures and is also due to phonon scattering. Our sample is polycrystalline and hence superconducting grains may be present which can form tunneling states. These states form the phonon scattering entities at low temperatures and hence the decrease in thermal diffusivity. The presence of the cusp in- 
dicates the importance of phonon-carrier interaction in the normal state of the Gd-doped YBCO samples. This effect is difficult to observe in conventional superconductors because of their large carrier concentration. We see that the cusp is the effect of the granular nature of the polycrystalline superconductor.

Due to the short coherence length $\xi$ of cuprate superconductors [for $\mathrm{YBa}_{2} \mathrm{Cu}_{3} \mathrm{O}_{7},{ }^{19} \xi_{a b}(0)=34 \AA, \xi_{c}(0)=7 \AA$ ], weaklink behavior was expected near $T_{c}$. Superconducting grains (here the term "grains" does not just imply physical grains but also superconducting portions separated by twin boundaries within physical grains) in polycrystalline samples start to couple weakly with their neighbors at $T_{c}$. At $T^{*}<T_{c}$ (Ref. 18) all the grains are coupled together and bulk superconductivity starts to occur. Once coupling between superconducting grains starts, the Gibb's free energy of the system must also include the coupling energy between grains. The inclusion of the coupling energy in the Gibb's free energy implies abrupt changes in the heat capacity, which varies as the second derivative of the total Gibb's free energy with respect to temperature. Such behavior was revealed by the cusp in the $\alpha_{s}-T$ curve at $T^{*}$, which coincides with the resistive transition offset temperature.

\section{ACKNOWLEDGMENTS}

This work was supported by the Research Grant Council of Hong Kong and a University of Hong Kong PostGraduate Student Grant.
${ }^{1}$ V. Z. Kresin and S. A. Wolf, Fundamentals of Superconductivity (Plenum, New York, 1990).

${ }^{2}$ Y. S. Song, H. K. Lee, and N. S. Chung, J. Appl. Phys. 65, 2568 (1989).

${ }^{3}$ Y. S. Song and N. S. Chung, J. Appl. Phys. 67, 935 (1990).

${ }^{4}$ K. C. Tsui, P. C. W. Fung, H. L. Tam, and G. O. Walker, J. Phys. Chem. Solids 52, 979 (1991).

${ }^{5}$ O. Pessoa, Jr., C. L. Cesar, N. A. Patel, H. Vargas, C. C. Ghizoni, and L. C. M. Miranda, J. Appl. Phys. 59, 1316 (1986).

${ }^{6}$ A. Rosencwaig and A. Gersho, J. Appl. Phys. 47, 64 (1975).

${ }^{7}$ P. Charpentier, F. Lepoutre, and L. Bertrand, J. Appl. Phys. 53, 608 (1982).

${ }^{8}$ F. A. McDonald and G. C. Wetsel, Jr., J. Appl. Phys. 49, 2313 (1978).

${ }^{9}$ G. Rousset, F. Lepoutre, and L. Bertrand, J. Appl. Phys. 54, 2383 (1983).

${ }^{10}$ L. K. T. Christopher, Photoacoustic Studies of Condensed Matter (unpublished).

${ }^{11}$ Z. Qi-Rui, Q. Yi-Tai, and C. Zu-Yao, Solid State Commun. 63, 415 (1987).

${ }^{12}$ H. G. A. Yuzhen, C. Genhua, and C. Liquan, Int. J. Mod. Phys. B 1, 199 (1987).

${ }^{13}$ G. A. West, J. J. Barrett, D. R. Siebert, and K. Virupaksha Reddy, Rev. Sci. Instrum. 54, 797 (1983).

${ }^{14}$ Fernelius, Appl. Opt. 18, 1784 (1979).

${ }^{15}$ J. Isaac, J. Philip, and B. K. Chaudhuri, Pramana Ind. J. Phys. 32, L167 (1989).

${ }^{16}$ M. Tinkham, Introduction to Superconductivity (McGraw-Hill, New York, 1975).

${ }^{17}$ N. E. Phillips, J. E. Emerson, and R. A. Fisher, J. Superconduct. 7, 251 (1994).

${ }^{18}$ C. E. Gough, IBM J. Res. Dev. 33, 262 (1989).

${ }^{19}$ T. K. Worthington, W. J. Gallagher, and T. R. Dinger, Phys. Rev. Lett. 59, 1160 (1987)

${ }^{20}$ L. Tewordt and Th. Wolkhausen, Solid State Commun. 70, 839 (1989).

${ }^{21}$ E. Eckert and R. Drake, Jr., Analysis of Heat and Mass Transfer (McGraw-Hill, Kogakusha, 1972).

${ }^{22}$ CRC Handbook of Chemistry and Physics, 72nd ed. (Chemical Rubber, Boca Raton, FL, 1991/92).

${ }^{23}$ S. B. Peralta, I. A. Vitkin, K. Ghandi, A. Mandelis, W. Sadowski, and E. Walker, in Photoacoustic and Photothermal Phenomena II, edited by J. C. Murphy (Springer, Berlin, 1990). 